\title{
Loss of Monoacylglycerol 0 Acyltransferase 2 Can Be Compensated for by Diacylglycerol 0 Acyltransferases 1 and 2 in High Fat Diet Induced
Obesity and Mammary Cancer Development
}

\section{Yan Mei}

Sun Yat-sen University Cancer Center

Jing Wang

Sun Yat-sen University Cancer Center

Jia-Bin Lu

Sun Yat-sen University Cancer Center

Guan-Ming Lu

Youjiang Medical University for Nationalities

Li-Xia Peng

Sun Yat-sen University Cancer Center

Yan-Hong Lang

Sun Yat-sen University Cancer Center

Li-Sheng Zheng

Sun Yat-sen University Cancer Center

Bi-Jun Huang

Sun Yat-sen University Cancer Center

Yan-Xia Shi

Sun Yat-sen University Cancer Center

Chao-Nan Qian ( $\nabla$ qianchn@sysucc.org.cn )

Sun Yat-sen University Cancer Center https://orcid.org/0000-0002-7233-8831

Research

Keywords: Mogat2 depletion, MMTV PyMT, mammary tumor, Dgat1, Dgat2

Posted Date: April 12th, 2021

DOl: https://doi.org/10.21203/rs.3.rs-398464/v1

License: (9) This work is licensed under a Creative Commons Attribution 4.0 International License.

Read Full License 
Page 2/9 


\section{Abstract}

Background: Dietary fat absorption involves the re esterification of digested triacylglycerol in the enterocytes, it is a biological process catalyzed by monoacylglycerol 0 acyltransferase 2 (MOGAT2, aka MGAT2), which is highly expressed in the small intestine. A previous study showed that the loss of the Mogat2 gene can prevent high fat diet induced obesity in mice. Obesity is associated with an increased risk of several types of cancer including postmenopausal breast cancer.

Methods: We collected 147 patients with triple negative breast adenocarcinoma to explore the relationship between the expression of MOGAT2 and patient overall survival. And we generated a Mogat2 deficient mouse mammary tumor model by crossing Mogat2 deficient mice with MMTV PyMT mice to examine the effect of losing MOGAT2 in vivo.

Results: Our founding suggest that obesity was induced by a relatively high fat diet ( $37 \%$ of calories from fat) in the mice with or without Mogat2 knockout. Mammary tumor development was deteriorated by a relatively high fat diet regardless of Mogat2 deficiency. As a compensation mechanism, upregulation of diacylglycerol 0 acyltransferases 1 and 2 (Dgat1 and Dgat2) in the Mogat2 deficient mice was found.

Conclusions: Elevated expression of MOGAT2 in triple negative breast adenocarcinoma predicts poorer patient overall survival. With the compensation of Dgat1 and Dgat2, Mogat2 deficiency alone cannot prevent fat diet induced obesity, nor prevent mammary tumor development in a mouse model.

\section{Full-text}

Due to technical limitations, full-text HTML conversion of this manuscript could not be completed. However, the manuscript can be downloaded and accessed as a PDF.

\section{Figures}



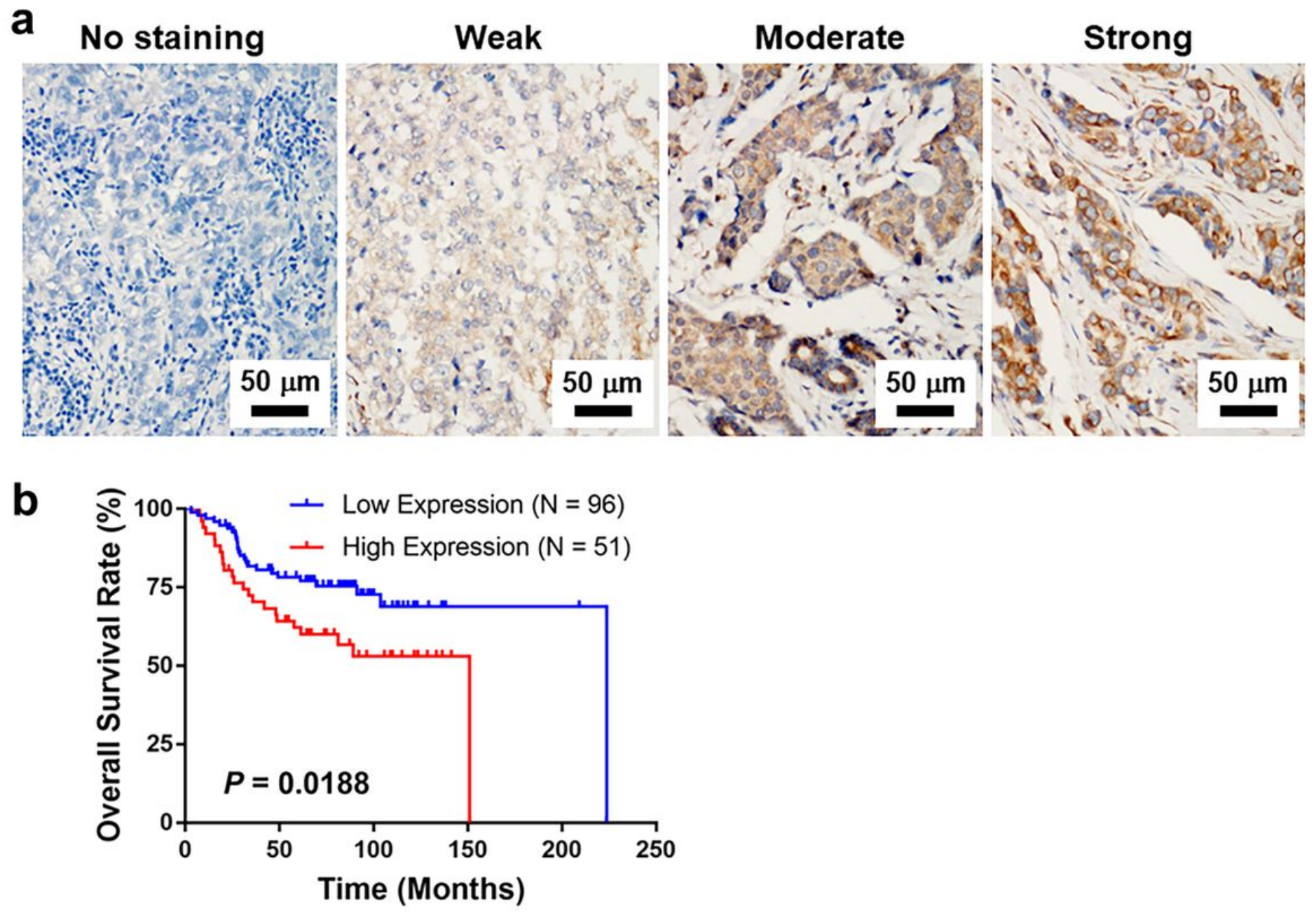

Figure 1

Figure 1

Primary tumor MOGAT2 expression level negatively correlates with poorer breast cancer patient survival. (a) Eight tissue arrays consisting of 147 triple negative breast cancer samples were constructed and immunohistochemically stained with a MOGAT2 antibody, followed by an assessment including the percentage of positively stained tumor cells and the intensity of staining on a 13-point scale. (b) Survival analyses showed that in the group with elevated MOGAT2 expression in the tumor, breast cancer patients had a poorer overall survival (OS) probability. 


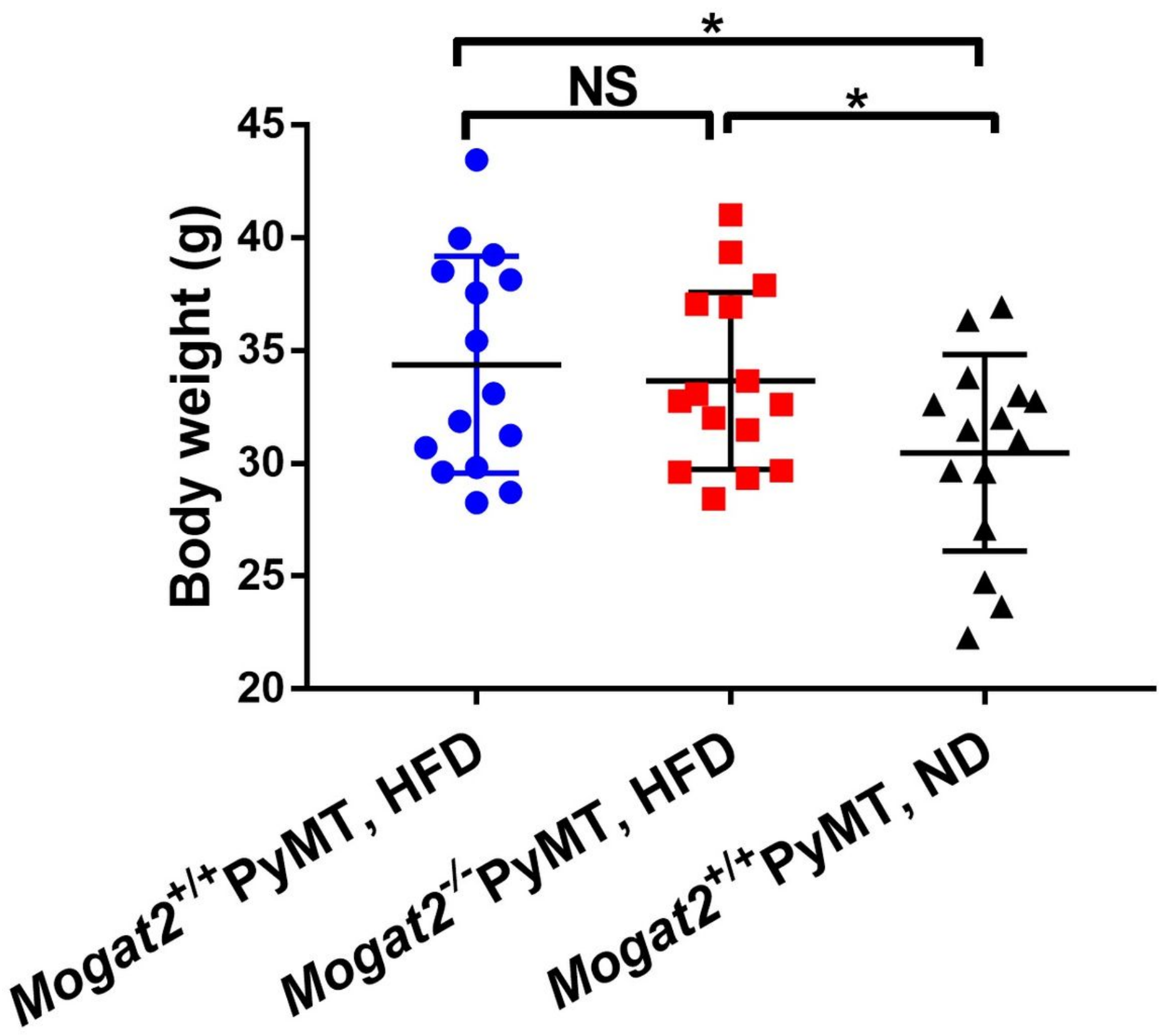

Figure 2

A relatively high fat diet promotes obesity regardless of Mogat2 depletion Body weight was determined in Mogat2+/+PyMT ( $n=15)$ and Mogat2-/-PyMT $(n=15)$ mice fed with a relatively high fat diet, as well as in the control group of Mogat2+/+PyMT $(n=15)$ fed with a normal diet. Increments in body weight were found to be independent of the Mogat2 gene. NS = non significant. 
a
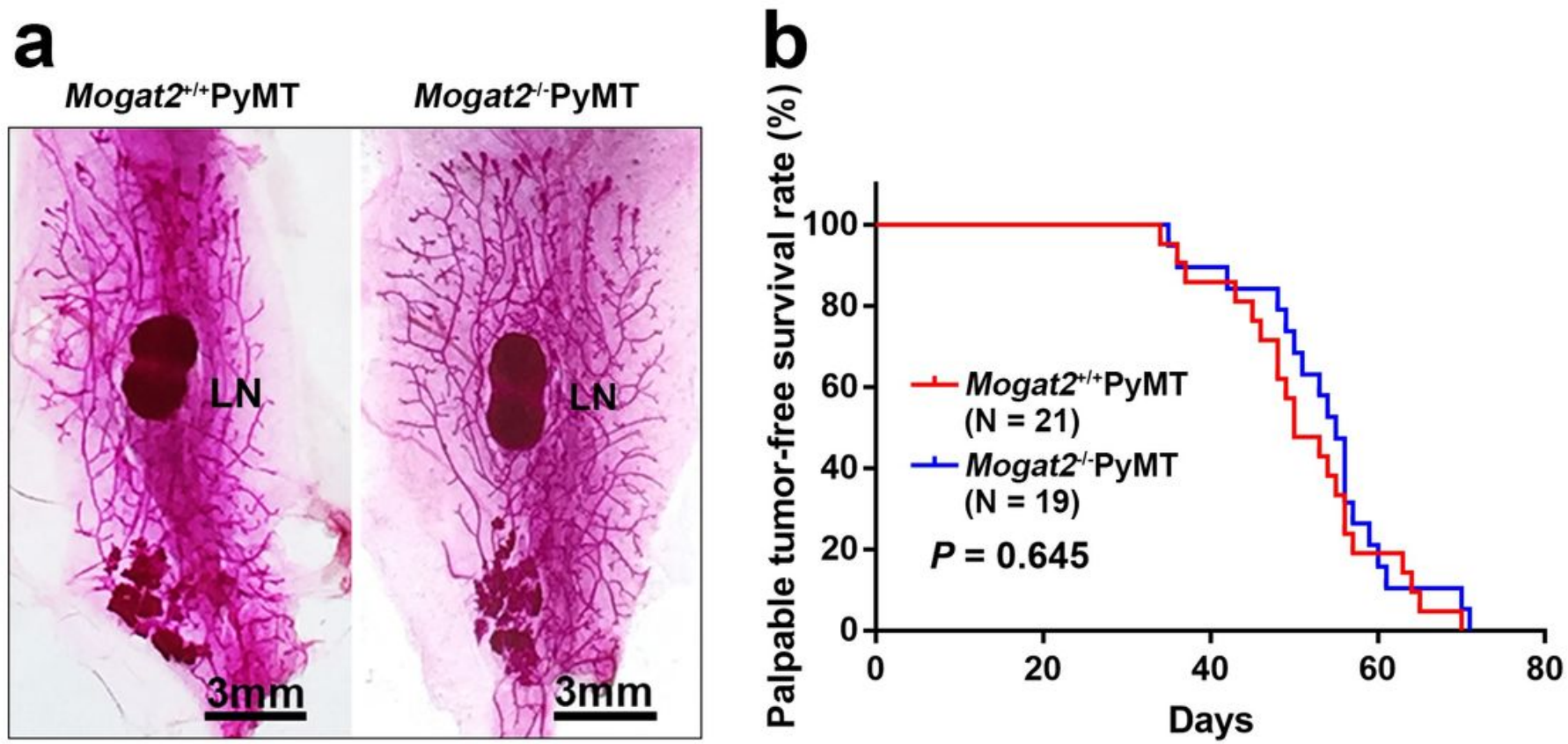

Figure 3

Mammary tumor initiation is not delayed by Mogat2 gene depletion. (a) Whole mount images of the fourth pair of mammary glands from Mogat2+/+PyMT and Mogat2-/-PyMT mice at 5 weeks old. LN = lymph node. (b) Kaplan Meier plots of the tumor latency of Mogat2+/+PyMT ( $n=19)$ and Mogat2-/ -PyMT $(n=21)$ mice. Data were analyzed by the log rank test. All mice were fed a normal diet $(10 \%$ of calories from fat). 


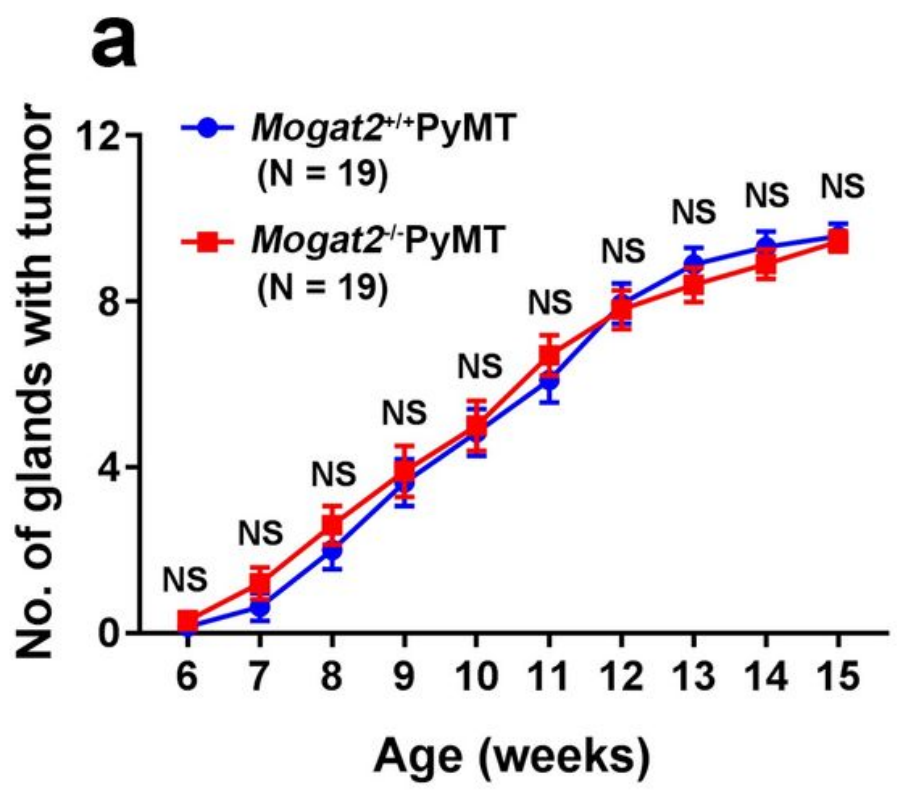

C

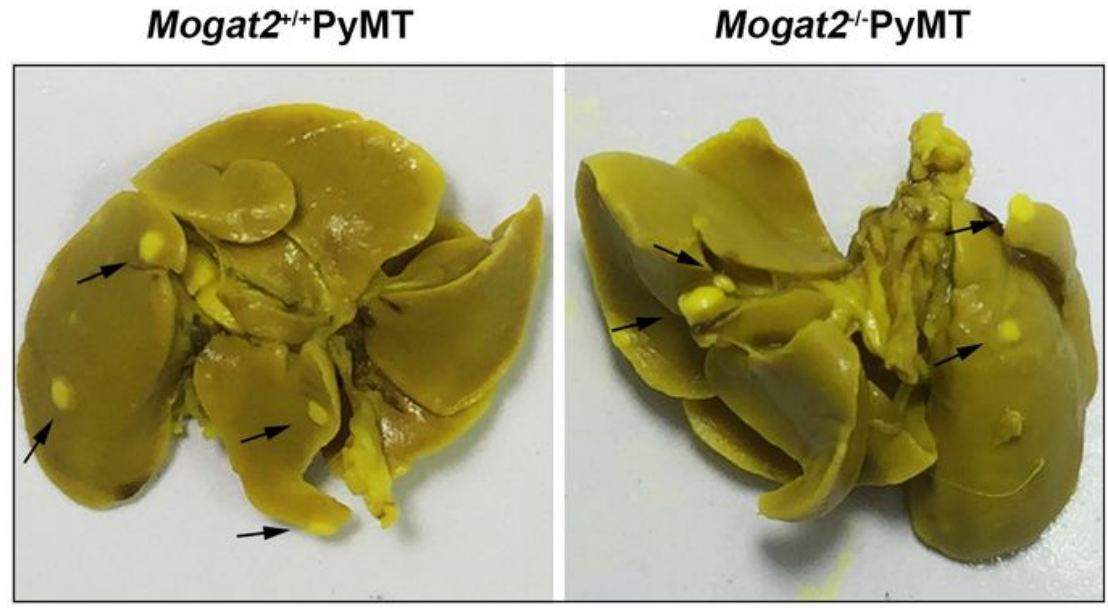

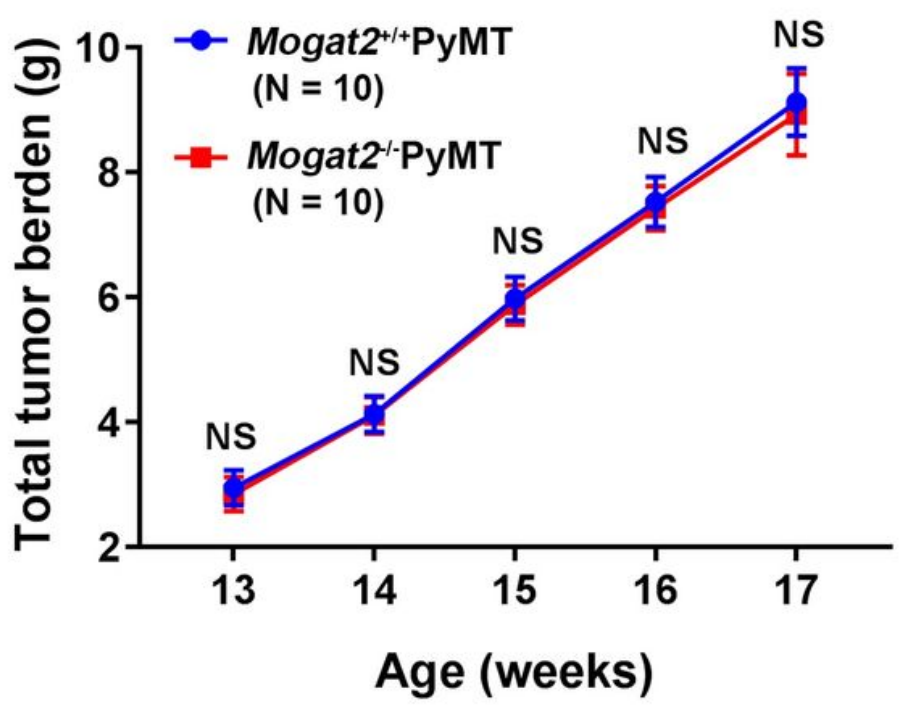

d

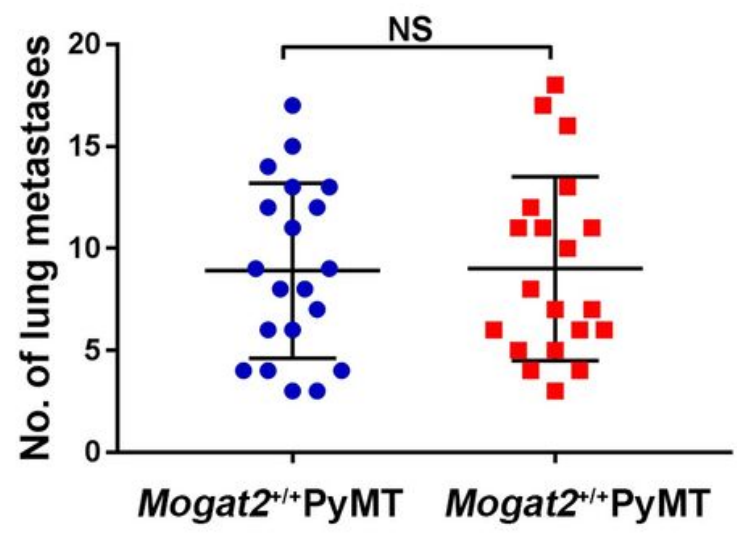

Figure 4

Mammary tumor progression is not inhibited by Mogat2 gene 415 depletion. (a) The number of mammary glands with palpable tumors for Mogat2+/+PyMT $(n=19)$ and Mogat2-/-PyMT $(n=19)$ mice. Data shown are the number of tumor bearing glands per mouse. Mammary tumors were monitored by weekly palpation. (b) Total tumor volume for Mogat2+/+PyMT $(n=10)$ and Mogat2-/-PyMT $(n=10)$ mice. (c--d) Lungs from Mogat2+/+PyMT ( $n=20)$ and Mogat2-/-PyMT ( $=20)$ mice were stained with Bouin's solution to identify metastases. Metastatic lesions are indicated by the arrows. (c) The data represents the mean total number of metastases \pm SEM from both groups at 15 weeks old (d) Data represents the mean \pm SEM, NS = non--significant. All mice were fed a normal diet. 

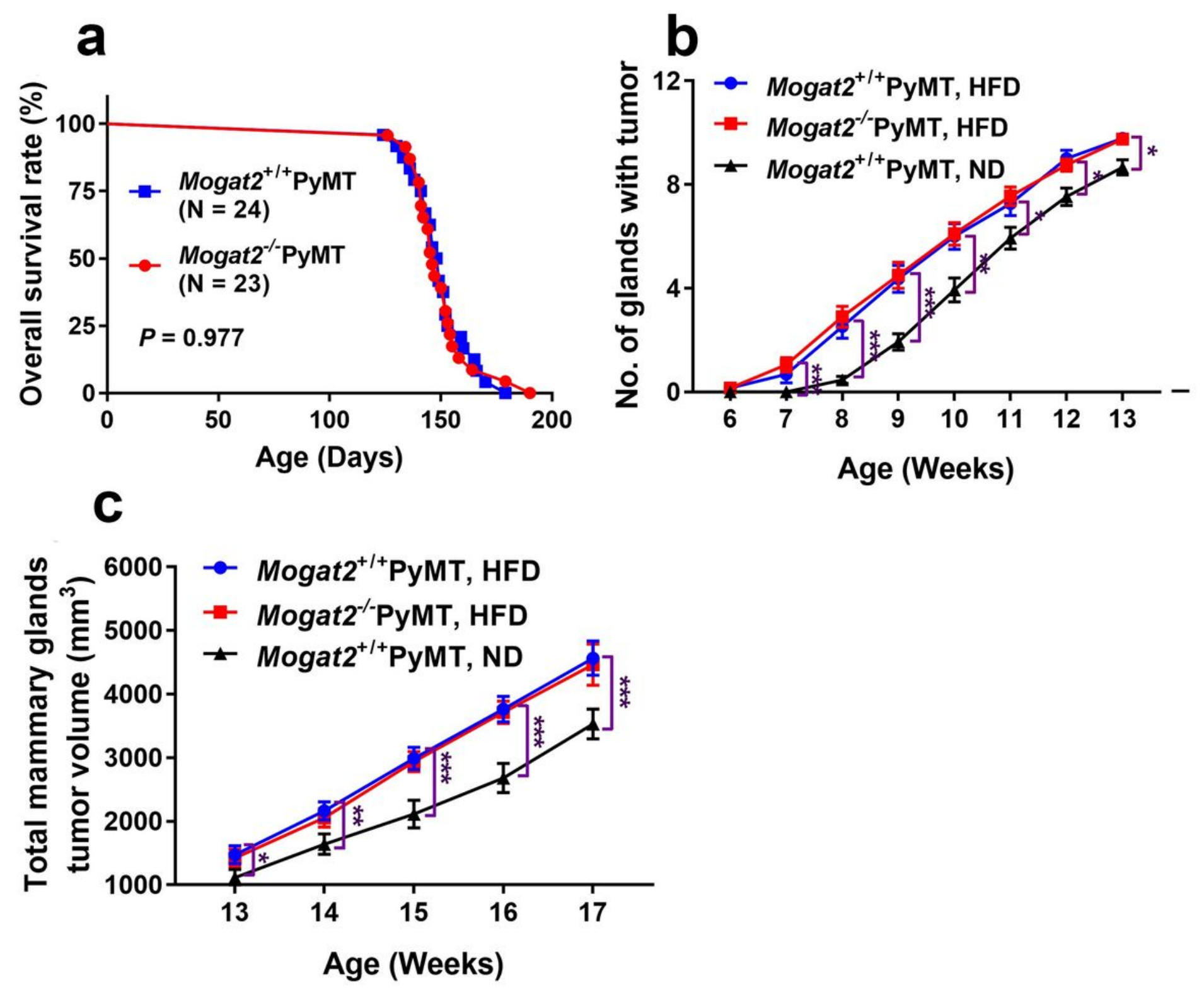

Figure 5

Mammary tumor progression is accelerated by a high fat diet regardless of Mogat2 gene depletion.

(a)Kaplan Meier curves showing the overall survival of Mogat2+/+PyMT ( $=24)$ and Mogat2-/-PyMT $(n=24)$ mice. Data were analyzed by the log rank test. The mice were fed on a normal diet. (b)The appearance of mammary tumors in Mogat2+/+PyMT $(n=19)$ and Mogat2-/-PyMT $(n=20)$ mice fed with a high fat diet (37\% of calories from fat), and Mogat2+/+PyMT $(n=15)$ fed on a normal diet were monitored by weekly palpation and are represented on Kaplan Meier curves. (c) The tumor volume of all mammary glands were determined in Mogat2+/+PyMT $(n=10)$ and Mogat2-/-PyMT $(n=10)$ mice fed on a high fat diet, as well as Mogat2+/+PyMT $(n=10)$ mice fed on a normal diet were measured weekly at 1317 weeks old. Data represent the mean \pm SEM. NS, non significant; HFD, high fat diet; ND, normal diet. $\star, P<0.05$. **, $P<0.01$. ***, $P<0.001$. 

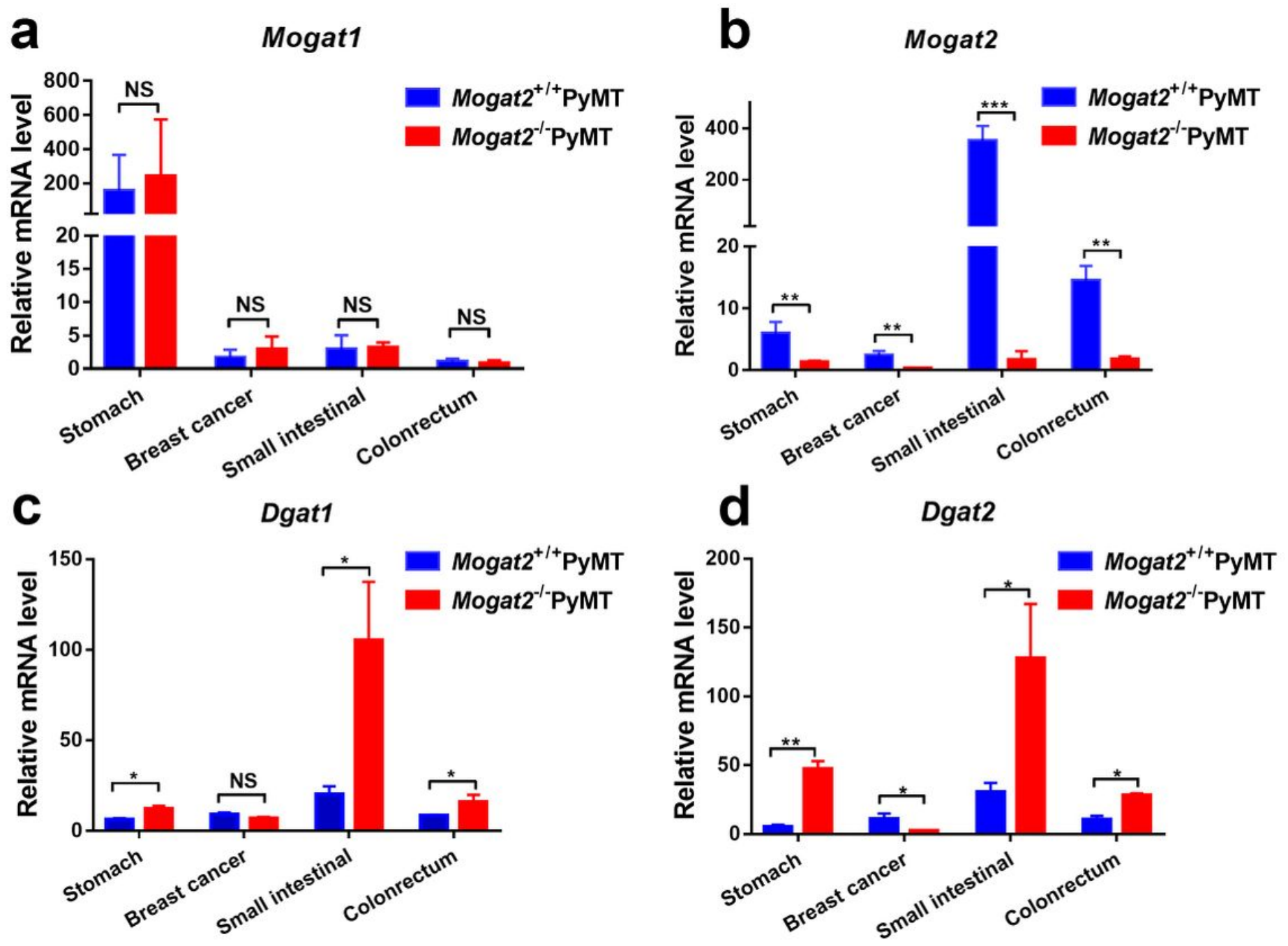

Figure 6

Depletion of the Mogat2 gene results in compensational overexpression of Dgat1 and Dgat2 genes. (a) Mogat1 gene mRNA was detected by qPCR in breast cancer, stomach, small intestine and colorectal tissues from Mogat2+/+PyMT $(n=6)$ and Mogat2-/-PyMT $(n=6)$ mice. As expected, Mogat1 gene expression was unaltered upon Mogat2 depletion. (b) In the same tissues, the Mogat2 expression level was suppressed in all the analyzed organs. (c) To compensate, Dgat1's mRNA level was upregulated in the gastrointestinal system, especially in the small intestine, which is the major organ for fat absorption. (d) The level of Dgat2 mRNA had a similar overexpression pattern with that of Dgat1. Data represent the mean $\pm S E M, N S=$ non significant, $* P<0.05, * * P<0.01$ and ${ }^{\star *} * \mathrm{P}<0.001$. 